\title{
Bargmann Symmetry Constraint and Binary Nonlinearization of Super Guo Hierarchy
}

\author{
Si-Xing Tao
}

\begin{abstract}
An explicit Bargmann symmetry constraint is computed and its associated binary nonlinearization of Lax pairs is carried out for the super Guo hierarchy. Under the obtained symmetry constraint, the n-th flow of the super Guo hierarchy is decomposed into two super finite -dimensional integrable Hamiltonian systems, which defined over the super symmetry manifold $R^{4 N \mid 2 N}$ with the corresponding dynamical variables $x$ and $t_{n}$. The integrals of motion required for Liouville integrability are explicitly given.
\end{abstract}

Index Terms - Symmetry constraint, binary nonlinearization, super guo hierarchy, super finitee-dimensional integrable hamiltonian systems.

\section{INTRODUCTION}

For almost twenty years, much attention has been paid to the construction of finite-dimensional integrable systems from soliton equations by using symmetry constraints. Either $(2+1)$-dimensional soliton equations [1], [2] or $(1+1)$ -dimensio -nal soliton equations [3], [4] can be decomposed into two compatible finite-dimensional integrable systems. It is known that a crucial idea in carrying out symmetry constraints is the nonlinearization of Lax pairs for soliton hierarchies. The nonlinearization of Lax pairs is classified into mono-nonlinearization [5]-[7] and binary nonlinearization [8], [9].

The technique of nonlinearization has been successfully applied to many well-known (1+1)-dimensional soliton equations, such as the AKNS hierarchy [3], the KdV hierarchy [4] and the Dirac hierarchy [10]. But there are few results on nonlinearization of super integrable systems, existing in the literature. But there are few results on nonlinearization of super integrable systems, existing in the literature. Studies provide many examples of super symmetry integrable systems, with super dependent variables and/or super independent variables [11]-[15]. Only very recently, nonlinearization were made for the super AKNS hierarchy, the super Dirac hierarchy and their corresponding super finite dimensional hierarchies were generated [16]-[18]. $\mathrm{Li}$ and Dong presented the super Hamiltonian structures of the super Guo hierarchy [19]. In this paper, we would like to consider the binary nonlinearization of the super Guo hierarchy.

This paper is organized as follows. In the next section, we

Manuscript received January 20, 2013; revised March 25, 2013. This work was supported by the National Natural Science Foundation of China under Grant No.61072147, the Natural Science Foundation of Henan Province under Grant No.132300410202, the Science and Technology Key Research Foundation of the Education Department of Henan Province under Grant No.12A110017 and the Youth Research Foundation of Shangqiu Normal University under Grant No. 2011QN12.

S.-X. Tao is with School of Mathematics and Information Science, Shangqiu Normal University, Henan, Shangqiu 476000, China (email: taosixing@163.com). will recall the super Guo soliton hierarchy and its super Hamiltonian structure. Then in Section III, we compute a Bargmann symmetry constraint for the potential of the super Guo hierarchy. In Section IV, we apply the binary nonlinearization method to super Guo hierarchy, and then obtain super finite-dimensional integrable Hamiltonian hierarchy on the super symmetry manifold $\mathbb{R}^{4 N \mid 2 N}$, whose integrals of motion are explicitly given.

\section{THE SUPER GuO HIERARCHY}

The super Guo spectral problem associated with Lie super algebra $B(0,1)$ is given by

$$
\begin{aligned}
\phi_{x}=U \phi, U & =\frac{1}{2}\left(\begin{array}{ccc}
\lambda^{-1} & q+r & \alpha \\
q-r & -\lambda^{-1} & \beta \\
\beta & -\alpha & 0
\end{array}\right), \\
U & =\left(\begin{array}{c}
q \\
r \\
\alpha \\
\beta
\end{array}\right), \phi=\left(\begin{array}{l}
\phi_{1} \\
\phi_{2} \\
\phi_{3}
\end{array}\right),
\end{aligned}
$$

where $\lambda$ is a spectral parameter, $q$ and $r$ are even variables, and $\alpha$ and $\beta$ are odd variables(see [20] ). Taking

$$
V=\frac{1}{2}\left(\begin{array}{ccc}
A & B+C & \rho \\
B-C & -A & \delta \\
\delta & -\rho & 0
\end{array}\right)
$$

the co-adjoint equation associated with (1) $V_{x}=[U, V]$ gives

$$
\left\{\begin{array}{c}
A_{x}=r B-q C+\frac{1}{2} \beta \rho+\frac{1}{2} \alpha \delta, \\
B_{x}=\lambda^{-1} C-r A-\frac{1}{2} \alpha \rho+\frac{1}{2} \beta \delta, \\
C_{x}=\lambda^{-1} B-q A-\frac{1}{2} \alpha \rho-\frac{1}{2} \beta \delta, \\
\rho_{x}=\frac{1}{2} \lambda^{-1} \rho-\frac{1}{2} \alpha A-\frac{1}{2} \beta B-\frac{1}{2} \beta C+\frac{1}{2} q \delta+\frac{1}{2} r \delta, \\
\delta_{x}=-\frac{1}{2} \lambda^{-1} \delta+\frac{1}{2} \beta A-\frac{1}{2} \alpha B+\frac{1}{2} \alpha C+\frac{1}{2} q \rho-\frac{1}{2} r \rho,
\end{array}\right.
$$

If we set

$$
A=\sum_{i \geq 0} A_{i} \lambda^{i}, B=\sum_{i \geq 0} B_{i} \lambda^{i}, C=\sum_{i \geq 0} C_{i} \lambda^{i}, \rho=\sum_{i \geq 0} \rho_{i} \lambda^{i},
$$

then (2) is equivalent to

$$
\left\{\begin{array}{c}
A_{i+1, x}=r B_{i+1}-q C_{i+1}+\frac{1}{2} \beta \rho_{i+1}+\frac{1}{2} \alpha \delta_{i+1}, \\
B_{i+1}=q A_{i}+C_{i x}+\frac{1}{2} \alpha \rho_{i}+\frac{1}{2} \beta \delta_{i}, \\
C_{i+1}=r A_{i}+B_{i x}+\frac{1}{2} \alpha \rho_{i}-\frac{1}{2} \beta \delta_{i}, \\
\rho_{i+1}=\alpha A_{i}+\beta B_{i}+\beta C_{i}+2 \rho_{i x}-q \delta_{i}-r \delta_{i}, \\
\delta_{i+1}=\beta A_{i}-\alpha B_{i}+\alpha C_{i}+q \rho_{i}-r \rho_{i}-2 \delta_{i x} .
\end{array}\right.
$$


which results in the following recurrence relations

$$
\left\{\begin{array}{c}
\left(B_{i+1},-C_{i+1}, \delta_{i+1},-\rho_{i+1}\right)^{T}=\mathcal{L}\left(B_{i},-C_{i}, \delta_{i},-\rho_{i}\right)^{T}, \\
\mathrm{~A}_{\mathrm{i}}=\partial^{-1}\left(r B_{i}-q C_{i}+\frac{1}{2} \beta \rho_{i}+\frac{1}{2} \alpha \delta_{i}\right), i \geq 0 .
\end{array}\right.
$$

where

$$
\mathcal{L}=\left(\begin{array}{cccc}
q \partial^{-1} r & -\partial+q \partial^{-1} q & L_{13} & L_{14} \\
-\partial-r \partial^{-1} r & -r \partial^{-1} q & L_{23} & L_{24} \\
-\alpha+\beta \partial^{-1} r & -\alpha+\beta \partial^{-1} q & L_{33} & L_{34} \\
-\beta-\alpha \partial^{-1} r & \beta-\alpha \partial^{-1} q & L_{43} & L_{43}
\end{array}\right)
$$

with $L_{13}=\frac{1}{2} \beta+\frac{1}{2} q \partial^{-1} \alpha, L_{14}=-\frac{1}{2} \alpha-\frac{1}{2} q \partial^{-1} \beta$,

$$
\begin{gathered}
L_{23}=\frac{1}{2} \beta-\frac{1}{2} r \partial^{-1} \alpha, L_{24}=\frac{1}{2} \alpha+\frac{1}{2} r \partial^{-1} \beta, \\
L_{33}=-2 \partial+\frac{1}{2} \beta \partial^{-1} \alpha, L_{34}=-\frac{1}{2} \beta \partial^{-1} \beta-q+r, \\
L_{13}=-\frac{1}{2} \alpha \partial^{-1} \alpha+q+r, L_{14}=2 \partial+\frac{1}{2} \alpha \partial^{-1} \beta .
\end{gathered}
$$

Upon choosing the initial conditions

$$
B_{0}=C_{0}=\rho_{0}=\delta_{0}=0, \quad A_{0}=1,
$$

all other $A_{i}, B_{i}, C_{i}, \rho_{i}, \delta_{i}(i \geq 1)$ can be worked out by the recurrence relations (5). The first few results are as follows:

$$
\begin{gathered}
A_{1}=0, B_{1}=q, C_{1}=r, \rho_{1}=\alpha, \delta_{1}=\beta, \\
A_{2}=-\frac{1}{2} q^{2}+\frac{1}{2} r^{2}-\alpha \beta, B_{2}=r_{x}, C_{2}=q_{x}, \rho_{2}=2 \alpha_{x}, \\
\delta_{2}=-2 \beta_{x}, A_{3}=q_{x} r-q r_{x}-2 \alpha_{x} \beta+2 \alpha \beta_{x}, \\
B_{3}=q_{x x}+\alpha \alpha_{x}-\beta \beta_{x}-\frac{1}{2} q^{3}+\frac{1}{2} q r^{2}-q \alpha \beta, \\
C_{3}=r_{x x}+\alpha \alpha_{x}+\beta \beta_{x}-\frac{1}{2} q^{2} r+\frac{1}{2} r^{3}-r \alpha \beta, \\
\rho_{3}=4 \alpha_{x x}+q_{x} \beta+2 q \beta_{x}+r_{x} \beta+2 r \beta_{x}-\frac{1}{2} q^{2} \alpha+\frac{1}{2} r^{2} \alpha, \\
\delta_{3}=4 \beta_{x x}+q_{x} \alpha+2 q \alpha_{x}-r_{x} \alpha-2 r \alpha_{x}-\frac{1}{2} q^{2} \beta+\frac{1}{2} r^{2} \beta .
\end{gathered}
$$

Let us associate the spectral problem (1) with the following auxiliary problem

$$
\phi_{t_{n}}=V^{(n)} \phi=\left(\lambda^{-n} V\right)_{-} \phi,
$$

with

$$
V^{(n)}=\sum_{i=0}^{n} \frac{1}{2}\left(\begin{array}{ccc}
A_{i} & B_{i}+C_{i} & \rho_{i} \\
B_{i}-C_{i} & -A_{i} & \delta_{i} \\
\delta_{i} & -\rho_{i} & 0
\end{array}\right) \lambda^{-n+i}
$$

where the minus symbol "-" denotes taking the non-positive part in the power of $\lambda$.

The compatible conditions of the spectral problem (1) and the auxiliary problem (7) are

$$
U_{t_{n}}-V_{x}^{(n)}+\left[U, V^{(n)}\right]=0,
$$

which infer the super Guo soliton hierarchy

$$
u_{t_{n}}=K_{n}=\left(C_{n+1}, B_{n+1}, \frac{1}{2} \rho_{n+1},-\frac{1}{2} \delta_{n+1}\right)^{T}, n \geq 0 .
$$

Here $u_{t_{n}}=K_{n}$ in (9) is called the $n$-th flow of this hierarchy,

When $\alpha=\beta=0$, the super integrable hierarchy (9) can be reduced to the hierarchy which was constructed by Guo in [22], so we call (9) as the super Guo hierarchy.

Using the super trace identity

$$
\frac{\delta}{\delta u} \int \operatorname{Str}\left(V \frac{\partial U}{\partial \lambda}\right) \mathrm{d} x=\left(\lambda^{-\gamma} \frac{\partial}{\partial \lambda} \lambda^{\gamma}\right) \operatorname{Str}\left(\frac{\partial U}{\partial u} V\right)
$$

where Str means the super trace [20,21], we have

$$
\left(\begin{array}{c}
B_{i+1} \\
-C_{i+1} \\
\delta_{i+1} \\
-\rho_{i+1}
\end{array}\right)=\frac{\delta}{\delta u} H_{i}, H_{i}=\int-\frac{A_{i+2}}{i+1} \mathrm{~d} x, i \geq 0 .
$$

Therefore, the super soliton hierarchy (9) can be written as the following super Hamiltonian form:

$$
u_{t_{n}}=J \frac{\delta H_{n}}{\delta u}
$$

where

$$
J=\left(\begin{array}{cccc}
0 & -1 & 0 & 0 \\
1 & 0 & 0 & 0 \\
0 & 0 & 0 & -\frac{1}{2} \\
0 & 0 & -\frac{1}{2} & 0
\end{array}\right)
$$

is a super symplectic operator, and $H_{n}$ is given by (11).

The first non-trivial nonlinear equations of the super Guo hierarchy (9) is given by its second flow

$$
\left\{\begin{array}{c}
q_{t_{2}}=r_{x x}+\alpha \alpha_{x}+\beta \beta_{x}-\frac{1}{2} q^{2} r+\frac{1}{2} r^{3}-r \alpha \beta \\
r_{t_{2}}=q_{x x}+\alpha \alpha_{x}-\beta \beta_{x}-\frac{1}{2} q^{3}+\frac{1}{2} q r^{2}-q \alpha \beta \\
\alpha_{t_{2}}=2 \alpha_{x x}+\frac{1}{2} q_{x} \beta+q \beta_{x}+\frac{1}{2} r_{x} \beta+r \beta_{x}-\frac{1}{4} q^{2} \alpha+\frac{1}{4} r^{2} \alpha \\
\beta_{t_{2}}=-2 \beta_{x x}-\frac{1}{2} q_{x} \alpha-q \alpha_{x}+\frac{1}{2} r_{x} \alpha+r \alpha_{x}+\frac{1}{4} q^{2} \beta-\frac{1}{4} r^{2} \beta
\end{array}\right.
$$

which possesses a Lax pair of $U$ and $V^{(2)}$ defined by

$$
V^{(2)}=\frac{1}{2}\left(\begin{array}{ccc}
\lambda^{-2}-\frac{1}{2} q^{2}+\frac{1}{2} r^{2}-\alpha \beta & V_{12}^{(2)} & V_{13}^{(2)} \\
(q-r) \lambda^{-1}-q_{x}+r_{x} & V_{22}^{(2)} & V_{23}^{(2)} \\
\beta \lambda^{-1}-2 \beta_{x} & V_{32}^{(2)} & 0
\end{array}\right),
$$

where

$$
\begin{gathered}
V_{12}^{(2)}=(q+r) \lambda^{-1}+q_{x}+r_{x}, V_{13}^{(2)}=\alpha \lambda^{-1}+2 \alpha_{x}, \\
V_{22}^{(2)}=-\lambda^{-2}+\frac{1}{2} q^{2}-\frac{1}{2} r^{2}+\alpha \beta, V_{23}^{(2)}=\beta \lambda^{-1}-2 \beta_{x}, \\
V_{32}^{(2)}=-\alpha \lambda^{-1}-2 \alpha_{x} .
\end{gathered}
$$

\section{BARGMANN SyMmETRY CONSTRAINT OF SUPER GUO} HIERARCHY

In order to compute a Bargmann symmetry constraint, we 
consider the following adjoint spectral problem of the spectral problem (1):

$\psi_{x}=-U^{S t} \psi=\frac{1}{2}\left(\begin{array}{ccc}-\lambda^{-1} & -q+r & \beta \\ -q-r & \lambda^{-1} & -\alpha \\ -\alpha & -\beta & 0\end{array}\right) \psi, \Psi=\left(\begin{array}{l}\psi_{1} \\ \psi_{2} \\ \psi_{3}\end{array}\right)$

where $S t$ means the super transposition. The following result is a general formula for the variational derivative with respect to the potential $u$ (see[3] for the classical case).

Lemma 1 [17]-[19] Let $U(u, \lambda)$ be an even matrix of order $m+n$ depending on $u, u_{x}, u_{x x}, \cdots$, and a parameter $\lambda$. Suppose that $\phi=\left(\phi_{e}, \phi_{o}\right)^{T}$ and $\psi=\left(\psi_{e}, \psi_{o}\right)^{T}$ satisfy the spectral problem and the adjoint spectral problem

$$
\phi_{x}=U(u, \lambda) \phi, \psi_{x}=-U^{S t} \psi
$$

where $\phi_{e}=\left(\phi_{1}, \cdots, \phi_{m}\right)$ and $\psi_{e}=\left(\psi_{1}, \cdots, \psi_{m}\right)$ are even eigenfunctions, and $\phi_{o}=\left(\phi_{m+1}, \cdots, \phi_{m+n}\right)$ and $\psi_{o}=$ $\left(\psi_{m+1}, \cdots, \psi_{m+n}\right)$ are odd eigenfunctions. Then the variational derivative of the parameter $\lambda$ with respect to the potential $\mathrm{u}$ is given by

$$
\frac{\delta \lambda}{\delta u}=\frac{\left(\psi_{e},(-1)^{p(u)} \psi_{o}\right)\left(\frac{\partial U}{\partial u}\right) \phi}{-\int \psi^{T}\left(\frac{\partial U}{\partial \lambda}\right) \phi \mathrm{d} x},
$$

where we denote

$$
p(v)=\left\{\begin{array}{r}
0, v \text { is an even variable }, \\
1, v \text { is an odd variable }
\end{array}\right.
$$

By Lemma 1, it is not difficult to find that

$$
\frac{\delta \lambda}{\delta u}=\frac{1}{2 E}\left(\begin{array}{c}
\psi_{1} \phi_{2}+\psi_{2} \phi_{1} \\
\psi_{1} \phi_{2}-\psi_{2} \phi_{1} \\
\psi_{1} \phi_{3}+\psi_{3} \phi_{2} \\
\psi_{2} \phi_{3}-\psi_{3} \phi_{1}
\end{array}\right)
$$

where $E=\int \frac{1}{2 \lambda^{2}}\left(\psi_{1} \phi_{1}-\psi_{2} \phi_{2}\right) \mathrm{d} x$. If we consider zero boundary conditions $\lim _{|x| \rightarrow \infty} \phi=\lim _{|x| \rightarrow \infty} \psi=0$, then we can obtain a characteristic property: a recurrence relation for the variational derivative of $\lambda$ :

$$
\mathcal{L} \frac{\delta \lambda}{\delta u}=\lambda \frac{\delta \lambda}{\delta u}
$$

where $\mathcal{L}$ and $\frac{\delta \lambda}{\delta u}$ are given by (5) and (19), respectively.

Let us now discuss the two spatial and temporal systems:

$$
\begin{aligned}
& \left(\begin{array}{l}
\phi_{1 j} \\
\phi_{2 j} \\
\phi_{3 j}
\end{array}\right)_{x}=U\left(u, \lambda_{j}\right)\left(\begin{array}{l}
\phi_{1 j} \\
\phi_{2 j} \\
\phi_{3 j}
\end{array}\right) \\
= & \frac{1}{2}\left(\begin{array}{ccc}
\lambda_{j}^{-1} & q+r & \alpha \\
q-r & -\lambda_{j}^{-1} & \beta \\
\beta & -\alpha & 0
\end{array}\right)\left(\begin{array}{l}
\phi_{1 j} \\
\phi_{2 j} \\
\phi_{3 j}
\end{array}\right), \\
& \left(\begin{array}{l}
\psi_{1 j} \\
\psi_{2 j} \\
\psi_{3 j}
\end{array}\right)_{x}=-U^{S t}\left(u, \lambda_{j}\right)\left(\begin{array}{l}
\psi_{1 j} \\
\psi_{2 j} \\
\psi_{3 j}
\end{array}\right)
\end{aligned}
$$

$$
=\frac{1}{2}\left(\begin{array}{ccc}
-\lambda_{j}^{-1} & -q+r & \beta \\
-q-r & \lambda_{j}^{-1} & -\alpha \\
-\alpha & -\beta & 0
\end{array}\right)\left(\begin{array}{l}
\psi_{1 j} \\
\psi_{2 j} \\
\psi_{3 j}
\end{array}\right)
$$

and

$$
\begin{aligned}
& \left(\begin{array}{l}
\phi_{1 j} \\
\phi_{2 j} \\
\phi_{3 j}
\end{array}\right)_{t_{n}}=V^{(n)}\left(u, \lambda_{j}\right)\left(\begin{array}{l}
\phi_{1 j} \\
\phi_{2 j} \\
\phi_{3 j}
\end{array}\right) \\
& =\frac{1}{2} \sum_{i=0}^{n} \lambda_{j}^{-n+i}\left(\begin{array}{ccc}
A_{i} & B_{i}+C_{i} & \rho_{i} \\
B_{i}-C_{i} & -A_{i} & \delta_{i} \\
\delta_{i} & -\rho_{i} & 0
\end{array}\right)\left(\begin{array}{c}
\phi_{1 j} \\
\phi_{2 j} \\
\phi_{3 j}
\end{array}\right), \\
& \left(\begin{array}{l}
\psi_{1 j} \\
\psi_{2 j} \\
\psi_{3 j}
\end{array}\right)_{t}=-\left(V^{(n)}\right)^{S t}\left(u, \lambda_{j}\right)\left(\begin{array}{c}
\psi_{1 j} \\
\psi_{2 j} \\
\psi_{3 j}
\end{array}\right) \\
& =\frac{1}{2} \sum_{i=0}^{n} \lambda_{j}^{-n+i}\left(\begin{array}{ccc}
-A_{i} & -B_{i}+C_{i} & \delta_{i} \\
-B_{i}-C_{i} & A_{i} & -\rho_{i} \\
-\rho_{i} & -\delta_{i} & 0
\end{array}\right)\left(\begin{array}{c}
\psi_{1 j} \\
\psi_{2 j} \\
\psi_{3 j}
\end{array}\right) \text {, }
\end{aligned}
$$

where $1 \leq j \leq N$ and $\lambda_{1}, \cdots, \lambda_{N}$ are $N$ distinct spectral parameters. Now for the system (21) and (22), we have the following symmetry constraints:

$$
\frac{\delta}{\delta u} H_{k}=\sum_{j=1}^{N} \gamma_{j} \frac{\delta \lambda_{j}}{\delta u}, k \geq 0 .
$$

The symmetry constraints in the case of $k=0$ is called a Bargmann constraint(see[9]). If taking $k=0, E_{j}=\gamma_{j}=$ $\int \frac{1}{2 \lambda_{j}^{2}}\left(\psi_{1 j} \phi_{1 j}-\psi_{2 j} \phi_{2 j}\right) \mathrm{d} x$, then it leads to an expression for the potential $u$, i.e.

$$
\left\{\begin{array}{c}
q=\frac{1}{2}\left(\left\langle\Psi_{1}, \Phi_{2}\right\rangle+\left\langle\Psi_{2}, \Phi_{1}\right\rangle\right), \\
r=\frac{1}{2}\left(\left\langle\Psi_{2}, \Phi_{1}\right\rangle-\left\langle\Psi_{1}, \Phi_{2}\right\rangle\right), \\
\alpha=-\frac{1}{2}\left(\left\langle\Psi_{2}, \Phi_{3}\right\rangle-\left\langle\Psi_{3}, \Phi_{1}\right\rangle\right), \\
\beta=\frac{1}{2}\left(\left\langle\Psi_{1}, \Phi_{3}\right\rangle+\left\langle\Psi_{3}, \Phi_{2}\right\rangle\right),
\end{array}\right.
$$

where we use the following notation

$$
\Phi_{i}=\left(\phi_{i 1}, \cdots, \phi_{i N}\right)^{T}, \Psi_{i}=\left(\Psi_{i 1}, \cdots, \Psi_{i N}\right)^{T}, i=1,2,3 .
$$

and $\langle\cdot, \cdot\rangle$ denotes the standard inner product of the Euclidean space $\mathbb{R}^{N}$.

\section{BINARY NONLINEARIZATION OF SUPER GUO HIERARCHY}

In this section, we want to perform binary nonlinearization for the Lax pairs and adjoint Lax pairs of super Guo hierarchy. To this end, let us substituting (24) into the Lax pairs and adjoint Lax pairs (21) and (22), and then we obtain the following nonlinearized Lax pairs and adjoint Lax pairs:

$$
\left(\begin{array}{l}
\phi_{1 j} \\
\phi_{2 j} \\
\phi_{3 j}
\end{array}\right)_{x}=U\left(\tilde{u}, \lambda_{j}\right)\left(\begin{array}{l}
\phi_{1 j} \\
\phi_{2 j} \\
\phi_{3 j}
\end{array}\right)
$$




$$
\begin{aligned}
= & \frac{1}{2}\left(\begin{array}{ccc}
\lambda_{j}^{-1} & \tilde{q}+\tilde{r} & \tilde{\alpha} \\
\tilde{q}-\tilde{r} & -\lambda_{j}^{-1} & \tilde{\beta} \\
\tilde{\beta} & -\tilde{\alpha} & 0
\end{array}\right)\left(\begin{array}{l}
\phi_{1 j} \\
\phi_{2 j} \\
\phi_{3 j}
\end{array}\right), \\
& \left(\begin{array}{l}
\psi_{1 j} \\
\psi_{2 j} \\
\psi_{3 j}
\end{array}\right)_{x}=-U^{S t}\left(\tilde{u}, \lambda_{j}\right)\left(\begin{array}{l}
\psi_{1 j} \\
\psi_{2 j} \\
\psi_{3 j}
\end{array}\right) \\
= & \frac{1}{2}\left(\begin{array}{ccc}
-\lambda_{j}^{-1} & -\tilde{q}+\tilde{r} & \tilde{\beta} \\
-\tilde{q}-\tilde{r} & \lambda_{j}^{-1} & -\tilde{\alpha} \\
-\tilde{\alpha} & -\tilde{\beta} & 0
\end{array}\right)\left(\begin{array}{l}
\psi_{1 j} \\
\psi_{2 j} \\
\psi_{3 j}
\end{array}\right),
\end{aligned}
$$

and

$$
\begin{gathered}
\left(\begin{array}{l}
\phi_{1 j} \\
\phi_{2 j} \\
\phi_{3 j}
\end{array}\right)_{t_{n}}=V^{(n)}\left(\tilde{u}, \lambda_{j}\right)\left(\begin{array}{c}
\phi_{1 j} \\
\phi_{2 j} \\
\phi_{3 j}
\end{array}\right) \\
=\frac{1}{2} \sum_{i=0}^{n} \lambda_{j}^{-n+i}\left(\begin{array}{ccc}
\tilde{A}_{i} & \tilde{B}_{i}+\tilde{C}_{i} & \tilde{\rho}_{i} \\
\tilde{B}_{i}-\tilde{C}_{i} & -\tilde{A}_{i} & \tilde{\delta}_{i} \\
\tilde{\delta}_{i} & -\tilde{\rho}_{i} & 0
\end{array}\right)\left(\begin{array}{l}
\phi_{1 j} \\
\phi_{2 j} \\
\phi_{3 j}
\end{array}\right), \\
\left(\begin{array}{l}
\psi_{1 j} \\
\psi_{2 j} \\
\psi_{3 j}
\end{array}\right)_{t}=-\left(V^{(n)}\right)^{S t}\left(\tilde{u}, \lambda_{j}\right)\left(\begin{array}{c}
\psi_{1 j} \\
\psi_{2 j} \\
\psi_{3 j}
\end{array}\right) \\
=\frac{1}{2} \sum_{i=0}^{n} \lambda_{j}^{-n+i}\left(\begin{array}{ccc}
-\tilde{A}_{i} & -\tilde{B}_{i}+\tilde{C}_{i} & \tilde{\delta}_{i} \\
-\tilde{B}_{i}-\tilde{C}_{i} & \tilde{A}_{i} & -\tilde{\rho}_{i} \\
-\tilde{\rho}_{i} & -\tilde{\delta}_{i} & 0
\end{array}\right)\left(\begin{array}{l}
\psi_{1 j} \\
\psi_{2 j} \\
\psi_{3 j}
\end{array}\right),
\end{gathered}
$$

where $1 \leq j \leq N$ and $\tilde{P}$ means an expression of $P(u)$ under the explicit constraint (24). Note that the spatial part of the nonlinearized system (25) is a system of ordinary differential equations with an independent variables $x$, but for a given $n(n \geq 2)$, the $t_{n}$-part of the nonlinearized system (26) is a system of ordinary differential equations. Obviously, the system (25) can be written as

$$
\begin{aligned}
& \Phi_{1, x}=\frac{1}{2} \Lambda^{-1} \Phi_{1}+\frac{1}{2}\left\langle\Psi_{2}, \Phi_{1}\right\rangle \Phi_{2} \\
&-\frac{1}{4}\left(\left\langle\Psi_{2}, \Phi_{3}\right\rangle-\left\langle\Psi_{3}, \Phi_{1}\right\rangle\right) \Phi_{3}, \\
& \Phi_{2, x}=\frac{1}{2}\left\langle\Psi_{1}, \Phi_{2}\right\rangle \Phi_{1}-\frac{1}{2} \Lambda^{-1} \Phi_{2}+\frac{1}{4}\left(\left\langle\Psi_{1}, \Phi_{3}\right\rangle+\left\langle\Psi_{3}, \Phi_{2}\right\rangle\right) \Phi_{3}, \\
& \Phi_{3, x}=\frac{1}{4}\left(\left\langle\Psi_{1}, \Phi_{3}\right\rangle\right.\left.+\left\langle\Psi_{3}, \Phi_{2}\right\rangle\right) \Phi_{1} \\
&+\frac{1}{4}\left(\left\langle\Psi_{2}, \Phi_{3}\right\rangle-\left\langle\Psi_{3}, \Phi_{1}\right\rangle\right) \Phi_{2}, \\
& \Psi_{1, x}=-\frac{1}{2} \Lambda^{-1} \Psi_{1}-\frac{1}{2}\left\langle\Psi_{1}, \Phi_{2}\right\rangle \Psi_{2}+\frac{1}{4}\left(\left\langle\Psi_{1}, \Phi_{3}\right\rangle+\left\langle\Psi_{3}, \Phi_{2}\right\rangle\right) \Psi_{3}, \\
& \Psi_{2, x}=-\frac{1}{2}\left\langle\Psi_{2}, \Phi_{1}\right\rangle \Psi_{1}+\frac{1}{2} \Lambda^{-1} \Psi_{2} \\
&+
\end{aligned}
$$

$$
\begin{aligned}
\Psi_{3, x}= & \frac{1}{4}\left(\left\langle\Psi_{2}, \Phi_{3}\right\rangle-\left\langle\Psi_{3}, \Phi_{1}\right\rangle\right) \Psi_{1} \\
& -\frac{1}{4}\left(\left\langle\Psi_{1}, \Phi_{3}\right\rangle+\left\langle\Psi_{3}, \Phi_{2}\right\rangle\right) \Psi_{2},
\end{aligned}
$$

where $\Lambda=\operatorname{diag}\left(\lambda_{1}, \cdots, \lambda_{m}\right)$. Then system (25) or (27) can be represented as the following Hamiltonian form:

$$
\left\{\begin{array}{c}
\Phi_{1, x}=\frac{\partial H_{1}}{\partial \Psi_{1}}, \Phi_{2, x}=\frac{\partial H_{1}}{\partial \Psi_{2}}, \Phi_{3, x}=\frac{\partial H_{1}}{\partial \Psi_{3}}, \\
\Psi_{1, x}=-\frac{\partial H_{1}}{\partial \Phi_{1}}, \Psi_{2, x}=-\frac{\partial H_{1}}{\partial \Phi_{2}}, \Psi_{3, x}=\frac{\partial H_{1}}{\partial \Phi_{3}} .
\end{array}\right.
$$

where

$$
\begin{aligned}
H_{1}= & \frac{1}{2}\left\langle\Lambda^{-1} \Psi_{1}, \Phi_{1}\right\rangle-\frac{1}{2}\left\langle\Lambda^{-1} \Psi_{2}, \Phi_{2}\right\rangle+\frac{1}{2}\left\langle\Psi_{2}, \Phi_{1}\right\rangle\left\langle\Psi_{1}, \Phi_{2}\right\rangle \\
& -\frac{1}{4}\left(\left\langle\Psi_{2}, \Phi_{3}\right\rangle-\left\langle\Psi_{3}, \Phi_{1}\right\rangle\right)\left(\left\langle\Psi_{1}, \Phi_{3}\right\rangle+\left\langle\Psi_{3}, \Phi_{2}\right\rangle\right) .
\end{aligned}
$$

When $n=1$, the system (26) is exactly the system (25) with $t_{1}=x$. When $n=2$, the system (26) is

$$
\begin{aligned}
& \Phi_{1, t_{2}}=\frac{1}{2}\left(\Lambda^{-2}-\frac{1}{2} \tilde{q}^{2}+\frac{1}{2} \tilde{r}^{2}-\tilde{\alpha} \tilde{\beta}\right) \Phi_{1} \\
&+\frac{1}{2}\left((\tilde{q}+\tilde{r}) \Lambda^{-1}+\tilde{q}_{x}+\tilde{r}_{x}\right) \Phi_{2}+\frac{1}{2}\left(\tilde{\alpha} \Lambda^{-1}+2 \tilde{\alpha}_{x}\right) \Phi_{3}, \\
& \Phi_{2, t_{2}}=\frac{1}{2}\left((\tilde{q}-\tilde{r}) \Lambda^{-1}-\tilde{q}_{x}+\tilde{r}_{x}\right) \Phi_{1} \\
&- \frac{1}{2}\left(\Lambda^{-2}-\frac{1}{2} \tilde{q}^{2}+\frac{1}{2} \tilde{r}^{2}-\tilde{\alpha} \tilde{\beta}\right) \Phi_{2}+\frac{1}{2}\left(\tilde{\beta} \Lambda^{-1}-2 \tilde{\beta}_{x}\right) \Phi_{3}, \\
& \Phi_{3, t_{2}}=\frac{1}{2}\left(\tilde{\beta} \Lambda^{-1}-2 \tilde{\beta}_{x}\right) \Phi_{1}-\frac{1}{2}\left(\tilde{\alpha} \Lambda^{-1}+2 \tilde{\alpha}_{x}\right) \Phi_{2}, \\
& \Psi_{1, t_{2}}=-\frac{1}{2}\left(\Lambda^{-2}-\frac{1}{2} \tilde{q}^{2}+\frac{1}{2} \tilde{r}^{2}-\tilde{\alpha} \tilde{\beta}\right) \Psi_{1} \\
&- \frac{1}{2}\left((\tilde{q}-\tilde{r}) \Lambda^{-1}-\tilde{q}_{x}+\tilde{r}_{x}\right) \Psi_{2}+\frac{1}{2}\left(\tilde{\beta} \Lambda^{-1}-2 \tilde{\beta}_{x}\right) \Psi_{3}, \\
& \Psi_{2, t_{2}}=-\frac{1}{2}\left((\tilde{q}+\tilde{r}) \Lambda^{-1}+\tilde{q}_{x}+\tilde{r}_{x}\right) \Psi_{1} \\
&+ \frac{1}{2}\left(\Lambda^{-2}-\frac{1}{2} \tilde{q}^{2}+\frac{1}{2} \tilde{r}^{2}-\tilde{\alpha} \tilde{\beta}\right) \Psi_{2}-\frac{1}{2}\left(\tilde{\alpha} \Lambda^{-1}+2 \tilde{\alpha}_{x}\right) \Psi_{3}, \\
& \Psi_{3, t_{2}}=-\frac{1}{2}\left(\tilde{\alpha} \Lambda^{-1}+2 \tilde{\alpha}_{x}\right) \Psi_{1}-\frac{1}{2}\left(\tilde{\beta} \Lambda^{-1}-2 \tilde{\beta}_{x}\right) \Psi_{2},(29)
\end{aligned}
$$

where $\tilde{q}, \tilde{r}, \tilde{\alpha}, \tilde{\beta}$ denote the functions $q, r, \alpha, \beta$ defined by the explicit constraint (24), and $\tilde{q}_{x}, \tilde{r}_{x}, \tilde{\alpha}_{x}, \tilde{\beta}_{x}$ are given by

$$
\begin{aligned}
\tilde{q}_{x}= & -\frac{1}{2}\left\langle\Lambda^{-1} \Psi_{1}, \Phi_{2}\right\rangle+\frac{1}{2}\left\langle\Lambda^{-1} \Psi_{2}, \Phi_{1}\right\rangle \\
& +\frac{1}{4}\left(\left\langle\Psi_{1}, \Phi_{2}\right\rangle-\left\langle\Psi_{1}, \Phi_{2}\right\rangle\right)\left(\left\langle\Psi_{1}, \Phi_{1}\right\rangle-\left\langle\Psi_{2}, \Phi_{2}\right\rangle\right), \\
\tilde{r}_{x}= & \frac{1}{2}\left\langle\Lambda^{-1} \Psi_{1}, \Phi_{2}\right\rangle+\frac{1}{2}\left\langle\Lambda^{-1} \Psi_{2}, \Phi_{1}\right\rangle \\
& -\frac{1}{4}\left(\left\langle\Psi_{1}, \Phi_{2}\right\rangle+\left\langle\Psi_{1}, \Phi_{2}\right\rangle\right)\left(\left\langle\Psi_{1}, \Phi_{1}\right\rangle-\left\langle\Psi_{2}, \Phi_{2}\right\rangle\right), \\
\tilde{\alpha}_{x}= & -\frac{1}{4}\left(\left\langle\Lambda^{-1} \Psi_{2}, \Phi_{3}\right\rangle-\left\langle\Lambda^{-1} \Psi_{3}, \Phi_{1}\right\rangle\right) \\
& +\frac{1}{8}\left(\left\langle\Psi_{1}, \Phi_{1}\right\rangle-\left\langle\Psi_{2}, \Phi_{2}\right\rangle\right)\left(\left\langle\Psi_{2}, \Phi_{2}\right\rangle-\left\langle\Psi_{3}, \Phi_{1}\right\rangle\right),
\end{aligned}
$$




$$
\begin{aligned}
\tilde{\beta}_{x}=- & \frac{1}{4}\left(\left\langle\Lambda^{-1} \Psi_{1}, \Phi_{3}\right\rangle+\left\langle\Lambda^{-1} \Psi_{3}, \Phi_{2}\right\rangle\right) \\
& +\frac{1}{8}\left(\left\langle\Psi_{1}, \Phi_{1}\right\rangle-\left\langle\Psi_{2}, \Phi_{2}\right\rangle\right)\left(\left\langle\Psi_{1}, \Phi_{3}\right\rangle+\left\langle\Psi_{3}, \Phi_{2}\right\rangle\right) .
\end{aligned}
$$

which are computed through using the spatial constrained flow (27). Then system (29) can be represented as the following super Hamiltonian form:

$$
\left\{\begin{array}{c}
\Phi_{1, x}=\frac{\partial H_{2}}{\partial \Psi_{1}}, \Phi_{2, x}=\frac{\partial H_{2}}{\partial \Psi_{2}}, \Phi_{3, x}=\frac{\partial H_{2}}{\partial \Psi_{3}} \\
\Psi_{1, x}=-\frac{\partial H_{2}}{\partial \Phi_{1}}, \Psi_{2, x}=-\frac{\partial H_{2}}{\partial \Phi_{2}}, \Psi_{3, x}=\frac{\partial H_{2}}{\partial \Phi_{3}}
\end{array}\right.
$$

where

$$
\begin{gathered}
H_{2}=\frac{1}{2}\left\langle\Lambda^{-2} \Psi_{1}, \Phi_{1}\right\rangle-\frac{1}{2}\left\langle\Lambda^{-2} \Psi_{2}, \Phi_{2}\right\rangle-\frac{1}{4}\left\langle\Psi_{2}, \Phi_{1}\right\rangle\left\langle\Psi_{1}, \Phi_{2}\right\rangle \cdot \\
\left(\left\langle\Psi_{1}, \Phi_{1}\right\rangle-\left\langle\Psi_{2}, \Phi_{2}\right\rangle\right)+\frac{1}{8}\left(\left\langle\Psi_{2}, \Phi_{3}\right\rangle-\left\langle\Psi_{3}, \Phi_{1}\right\rangle\right) \cdot \\
\left(\left\langle\Psi_{1}, \Phi_{3}\right\rangle+\left\langle\Psi_{3}, \Phi_{2}\right\rangle\right)\left(\left\langle\Psi_{1}, \Phi_{1}\right\rangle-\left\langle\Psi_{2}, \Phi_{2}\right\rangle\right)+ \\
\frac{1}{2}\left\langle\Psi_{2}, \Phi_{1}\right\rangle\left\langle\Lambda^{-1} \Psi_{1}, \Phi_{2}\right\rangle+\frac{1}{2}\left\langle\Lambda^{-1} \Psi_{2}, \Phi_{1}\right\rangle\left\langle\Psi_{1}, \Phi_{2}\right\rangle \\
-\frac{1}{4}\left(\left\langle\Psi_{2}, \Phi_{3}\right\rangle-\left\langle\Psi_{3}, \Phi_{1}\right\rangle\right)\left(\left\langle\Lambda^{-1} \Psi_{1}, \Phi_{3}\right\rangle+\left\langle\Lambda^{-1} \Psi_{3}, \Phi_{2}\right\rangle\right) \\
-\frac{1}{4}\left(\left\langle\Lambda^{-1} \Psi_{2}, \Phi_{3}\right\rangle-\left\langle\Lambda^{-1} \Psi_{3}, \Phi_{1}\right\rangle\right)\left(\left\langle\Psi_{1}, \Phi_{3}\right\rangle+\left\langle\Psi_{3}, \Phi_{2}\right\rangle\right) .
\end{gathered}
$$

In addition, the characteristic property (20) and the recurrence relations (5) ensure that

$$
\left\{\begin{array}{c}
\tilde{A}_{i+1}=\frac{1}{2}\left(\left\langle\Lambda^{-i} \Psi_{1}, \Phi_{1}\right\rangle-\left\langle\Lambda^{-i} \Psi_{2}, \Phi_{2}\right\rangle\right), i \geq 0 \\
\tilde{B}_{i+1}=\frac{1}{2}\left(\left\langle\Lambda^{-i} \Psi_{1}, \Phi_{2}\right\rangle+\left\langle\Lambda^{-i} \Psi_{2}, \Phi_{1}\right\rangle\right), i \geq 0 \\
\tilde{C}_{i+1}=\frac{1}{2}\left(\left\langle\Lambda^{-i} \Psi_{2}, \Phi_{1}\right\rangle-\left\langle\Lambda^{-i} \Psi_{1}, \Phi_{2}\right\rangle\right), i \geq 0 \\
\tilde{\rho}_{i+1}=-\frac{1}{2}\left(\left\langle\Lambda^{-i} \Psi_{2}, \Phi_{3}\right\rangle-\left\langle\Lambda^{-i} \Psi_{3}, \Phi_{1}\right\rangle\right), i \geq 0 \\
\tilde{\delta}_{i+1}=\frac{1}{2}\left(\left\langle\Lambda^{-i} \Psi_{1}, \Phi_{3}\right\rangle+\left\langle\Lambda^{-i} \Psi_{3}, \Phi_{2}\right\rangle\right), i \geq 0
\end{array}\right.
$$

Then the co-adjoint representation equation $\tilde{V}_{x}=[\widetilde{U}, \tilde{V}]$ remains true. Furthermore, we know that $\tilde{V}_{x}^{2}=\left[\widetilde{U}, \widetilde{V}_{x}^{2}\right]$ is also true. Let

$$
F=\frac{1}{4} \operatorname{Str} V^{2}
$$

Then it is easy to find that $F_{x}=0$. That is to say, $F$ is a generating function of integrals of motion for the system (25) or (27). Due to $F=\sum_{n \geq 0} F_{n} \lambda^{n}$, we obtain the following formulas of integrals of motion:

$$
\begin{gathered}
F_{0}=\frac{1}{2} \tilde{A}_{0}^{2}, F_{1}=\tilde{A}_{0} \tilde{A}_{1}, \\
F_{n}=\tilde{A}_{0} \tilde{A}_{n}+\frac{1}{2} \sum_{i=1}^{n-1}\left(\tilde{A}_{i} \tilde{A}_{n-i}+\tilde{B}_{i} \tilde{B}_{n-i}-\tilde{C}_{i} \tilde{C}_{n-i}\right. \\
\left.+2 \tilde{\rho}_{i} \tilde{\delta}_{n-i}\right), n \geq 2 .
\end{gathered}
$$

Substituting (32) into the above formulas of integrals of motion, we obtain the following expression of $F_{m}(m \geq 0)$ :

$$
\begin{gathered}
F_{0}=\frac{1}{2}, F_{1}=\frac{1}{2}\left(\left\langle\Psi_{1}, \Phi_{1}\right\rangle-\left\langle\Psi_{2}, \Phi_{2}\right\rangle\right), \\
F_{2}=\frac{1}{2}\left\langle\Lambda^{-1} \Psi_{1}, \Phi_{1}\right\rangle-\frac{1}{2}\left\langle\Lambda^{-1} \Psi_{2}, \Phi_{2}\right\rangle+\frac{1}{2}\left\langle\Psi_{2}, \Phi_{1}\right\rangle\left\langle\Psi_{1}, \Phi_{2}\right\rangle
\end{gathered}
$$

$$
\begin{gathered}
-\frac{1}{4}\left(\left\langle\Psi_{2}, \Phi_{3}\right\rangle-\left\langle\Psi_{3}, \Phi_{1}\right\rangle\right)\left(\left\langle\Psi_{1}, \Phi_{3}\right\rangle+\left\langle\Psi_{3}, \Phi_{2}\right\rangle\right), \\
F_{n}=\frac{1}{2}\left\langle\Lambda^{-n+1} \Psi_{1}, \Phi_{1}\right\rangle-\frac{1}{2}\left\langle\Lambda^{-n+1} \Psi_{2}, \Phi_{2}\right\rangle+ \\
\sum_{i=1}^{n-1} \frac{1}{8}\left(\left\langle\Lambda^{-i+1} \Psi_{1}, \Phi_{1}\right\rangle-\left\langle\Lambda^{-i+1} \Psi_{2}, \Phi_{2}\right\rangle\right) \times \\
\left(\left\langle\Lambda^{-n+i+1} \Psi_{1}, \Phi_{1}\right\rangle-\left\langle\Lambda^{-n+i+1} \Psi_{2}, \Phi_{2}\right\rangle\right)+ \\
\sum_{i=1}^{n-1} \frac{1}{4}\left(\left\langle\Lambda^{-i+1} \Psi_{1}, \Phi_{2}\right\rangle\left\langle\Lambda^{-n+i+1} \Psi_{2}, \Phi_{1}\right\rangle\right. \\
\left.\quad\left\langle\Lambda^{-i+1} \Psi_{2}, \Phi_{1}\right\rangle\left\langle\Lambda^{-n+i+1} \Psi_{1}, \Phi_{2}\right\rangle\right) \\
-\sum_{i=1}^{n-1} \frac{1}{4}\left(\left\langle\Lambda^{-i+1} \Psi_{2}, \Phi_{3}\right\rangle-\left\langle\Lambda^{-i+1} \Psi_{3}, \Phi_{1}\right\rangle\right) \times \\
\left(\left\langle\Lambda^{-n+i+1} \Psi_{1}, \Phi_{3}\right\rangle+\left\langle\Lambda^{-n+i+1} \Psi_{3}, \Phi_{2}\right\rangle\right), n \geq 3 .
\end{gathered}
$$

On the other hand, let us consider the temporal part of nonlinearized system (26). Making use of (32) and (35), the system (26) can be represented as the following super Hamiltonian form:

$$
\left\{\begin{array}{c}
\Phi_{1, t_{n}}=\frac{\partial F_{n+1}}{\partial \Psi_{1}}, \Phi_{2, t_{n}}=\frac{\partial F_{n+1}}{\partial \Psi_{2}}, \Phi_{3, t_{n}}=\frac{\partial F_{n+1}}{\partial \Psi_{3}}, \\
\Psi_{1, t_{n}}=-\frac{\partial F_{n+1}}{\partial \Phi_{1}}, \Psi_{2, t_{n}}=-\frac{\partial F_{n+1}}{\partial \Phi_{2}}, \Psi_{3, t_{n}}=\frac{\partial F_{n+1}}{\partial \Phi_{3}} .
\end{array}\right.
$$

This can be checked pretty easily. For example, we can show one equality in the above system as follows:

$$
\begin{gathered}
\Psi_{2, t_{n}}=-\sum_{i=0}^{n} \frac{1}{2}\left(\tilde{B}_{i}+\tilde{C}_{i}\right) \Lambda^{-n+i} \Psi_{1}+\sum_{i=0}^{n} \frac{1}{2} A_{i} \Lambda^{-n+i} \Psi_{2}- \\
\sum_{i=0}^{n} \frac{1}{2} \rho_{i} \Lambda^{-n+i} \Psi_{3} \\
=-\sum_{i=0}^{n} \frac{1}{2}\left\langle\Lambda^{-i+1} \Psi_{1}, \Phi_{2}\right\rangle \Lambda^{-n+i} \Psi_{1}+ \\
\sum_{i=0}^{n} \frac{1}{4}\left(\left\langle\Lambda^{-i+1} \Psi_{1}, \Phi_{1}\right\rangle-\left\langle\Lambda^{-i+1} \Psi_{2}, \Phi_{2}\right\rangle\right) \Lambda^{-n+i} \Psi_{2} \\
+\frac{1}{2} \Lambda^{-n+1} \Psi_{2}+\sum_{i=0}^{n} \frac{1}{4}\left(\left\langle\Lambda^{-i+1} \Psi_{1}, \Phi_{1}\right\rangle\right. \\
\left.-\left\langle\Lambda^{-i+1} \Psi_{2}, \Phi_{2}\right\rangle\right) \Lambda^{-n+i} \Psi_{3} \\
=-\frac{\partial F_{n+1}}{\partial \Phi_{2}} .
\end{gathered}
$$

In order to show the Liouville integrability for the constrained flows (25) and (26), we need to prove the commutative propertity of motion $\left\{F_{m}\right\}_{m \geq 0}$, under the corresponding Poission bracket

$$
\begin{gathered}
\{F, G\}=\sum_{i=1}^{3} \sum_{j=1}^{N}\left(\frac{\partial F}{\partial \phi_{i j}} \frac{\partial G}{\partial \psi_{i j}}\right. \\
\left.-(-1)^{p\left(\phi_{i j}\right)}(-1)^{p\left(\psi_{i j}\right)} \frac{\partial F}{\partial \phi_{i j}} \frac{\partial G}{\partial \psi_{i j}}\right) .
\end{gathered}
$$

At this time, we still have an equality $\tilde{V}_{t_{n}}=\left[\tilde{V}^{(n)}, \tilde{V}\right]$, and After a similar discussion, we know that $F_{m}(m \geq 0)$ are integrals of motion for the system (26) or (36), which implies 


$$
\left\{F_{m+1}, F_{n+1}\right\}=\frac{\partial}{\partial t_{n}} F_{m+1}=0, m, n \geq 0 .
$$

The above equality (39) shows that $F_{m}(m \geq 0)$ are in involution in pair under the Poisson bracket (38).

In addition, similar to [16], we know that

$$
f_{k}=\psi_{1 k} \phi_{1 k}+\psi_{2 k} \phi_{2 k}+\psi_{3 k} \phi_{3 k}, 1 \leq k \leq N
$$

are integrals of motion for (25) and (26). It is not difficult to verify that $3 N$ functions $\left\{F_{m}\right\}_{m=1}^{2 N}$ and $\left\{f_{k}\right\}_{k=1}^{N}$ are involution in pair. To show the functional independence of the $3 N$ functions $\left\{F_{m}\right\}_{m=1}^{2 N}$ and $\left\{f_{k}\right\}_{k=1}^{N}$, we can use the method in [16], or the technique by ma etal[23,24]. Therefore, the $3 N$ functions $\left\{F_{m}\right\}_{m=1}^{2 N}$ and $\left\{f_{k}\right\}_{k=1}^{N}$, are functional independent over some region of the super symmetry manifold $\mathbb{R}^{4 N \mid 2 N}$. Now, all of the above analysis gives the following theorem.

Theorem 1 Both the spatial and temporal flows (25) and (26) are Liouville integrable Hamiltonian systems defined on the super symmetry manifold $\mathbb{R}^{4 N \mid 2 N}$, which possess $3 N$ functionally independent and involutive integrals of motion $\left\{F_{m}\right\}_{m=1}^{2 N}$ and $\left\{f_{k}\right\}_{k=1}^{N}$ defined by (35) and (40).

\section{REFERENCES}

[1] Y. Cheng and Y. S. Li, "The constraint of the Kadomtsev-Petviashvili equation and its special solutions," Phys. Lett. A, vol. 157, no. 1, pp. 22 -26 , July 1991

[2] Y. Cheng, "Constraints of the Kadomtsev-Petviashvili Hierarchy," J. Math. Phys., vol. 33, no. 11, pp. 3774-3782, Nov. 1992.

[3] W. X. Ma and W. Strampp, "An explicit symmetry constraint for the Lax pairs of AKNS systems," Phys. Lett. A, vol. 185, no. 3, pp. 277-286, Feb. 1994.

[4] W. X. Ma, "New finite-dimensional integrable systems by symmetry constraint of the KdV equations," J. Phys. Soc. Jpn., vol. 64, no. 4, pp. 1085-1091, April 1995.

[5] Y. B. Zeng and Y. S. Li, "The constraints of potentials and the finite dimensional integrable systems," J. Math. Phys., vol. 30, no. 8, pp. 1679 -1689, Aug. 1989.

[6] C. W. Cao, "Nonlinearization of the Lax system for AKNS Hierarchy," Sci. China Ser. A, vol. 33, no.5, pp. 528-536, May 1990.

[7] C. W. Cao and X. G. Geng, "A monconfocal generator of involutive systems and three associated soliton hierarchies," J. Math. Phys., vol. 32, no. 9, pp. 2323-2328, Sep. 1991.

[8] W. X. Ma, "Symmetry constraint of MKdV equations by binary nonlinearization," Phys. A, vol. 219, no. 3-4, pp. 467-481, Oct. 1995.

[9] W. X. Ma and R. G. Zhou, "Adjoint symmetry constraints leading to binary nonlinearization,” J. Nonlinear Math. Phys., vol. 9, no. Suppl. 1, pp. 106-126, Feb. 2002.
[10] W. X. Ma, "Binary nonlinearization for the Dirac systems," Chin. Ann. Math., vol. 18B, no. 1, pp. 79-88, Jan. 1997.

[11] Z. Popowicz, "The fully supersymmetric AKNS equations," J. Phys. A: Math. Gen., vol. 23, no. 7, pp. 1127-1136, April 1990.

[12] M. Gürses and Ö. Oğuz, "A super AKNS scheme," Phys. Lett. A, vol. 108, no. 9, pp. 437-440, April 1985.

[13] Q. P. Liu and M. Ma $\ddot{n}$ as, "Darboux transformation for super-symmetric KP hierarchies," Phys. Lett. B, vol. 485, no. 1-3, pp. 293-300, July 2000.

[14] B. A. Kupershmidt, "A super Korteweg-de Vries equation: an integrable system,” Phys. Lett. A, vol. 102, no. 5-6, pp. 213-215, May 1984.

[15] Y.S. Li and L. N. Zhang, "Hamiltonian structure of the super evolution equation,” J. Math. Phys., vo1. 31, no. 2, pp. 470-475, Feb. 1990.

[16] J. S. He, J. Yu, Y. Cheng, and R. G. Zhou, "Binary nonlinearization of the super AKNS system," Modern Phys. Lett. B, vol. 22, no. 4, pp. 275 - 288, Feb. 2008

[17] J. Yu, J. W. Han, and J. S. He, "Binary nonlinearization of the super AKNS system under an implicit symmetry constraint," J. Phys. A: Math. Theo., vol. 42, no. pp. 465201, Nov. 2009.

[18] J. Yu, J. S. He, W. X. Ma, and Y. Cheng, "The Bargmann symmetry constraint and binary nonlinearization of the super Dirac system," Chin. Ann. Math., vol. 31B, no. 3, pp. 361-372, May 2010.

[19] Z. Li, H. H. Dong, and H. H. Yang, "A super-soliton hierarchy and its super-Hamiltonian structure," Int. J. Theor. Phys., vol. 48, no. 7, pp. 2172 -2176, July 2009.

[20] W. X. Ma, J. S. He, and Z. Y. Qin, "A supertrace identity and its applications to super integrable systems," J. Math. Phys., vol. 49, no. 3, pp. 033511, Mar. 2008.

[21] X. B. Hu, "An approach to generate superextensions of integrable systems," J. Phys. A: Math. Gen., vol. 30, no. 2, pp. 619-632, Jan. 1997.

[22] F. K. Guo, "Two Hierarcihes of Integrable Hamiltonian Equations, " Mathematica Applicata, vol. 9, no. 4 , pp. 495-499, Oct. 1996.

[23] W. X. Ma, B. Fuchssteiner, and W. Oevel, "A $3 \times 3$ matrix spectral problem for AKNS hierarchy and its binary nonlinearization," Phys. A, vol. 233, no. 1-2, pp. 331-354, Nov. 1996

[24] W. X. Ma and Z. X. Zhou, "Binary symmetry constraints of $N$-wave intersection equations in $1+1$ and $2+1$ dimensions," J. Math. Phys., vol. 42 ,no. 9, pp. 4345-4382, Sep. 2001.

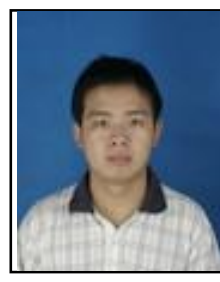

Si-Xing Tao was born in March 1981, in Qingzhou, Shandong Province of China. He received the bachelor degree in Mathematics and Applied Mathematics from Qufu Normal University in 2003 and the master degree in Basic Mathematics from Qufu Normal University in 2006. He received the doctor degree in Basic Mathematics from Shanghai University in 2011. Now he is a lecturer in School of Mathematics and Information Science, Shangqiu Normal University. His research interests are in soliton theory and integrable systems and published more than 10 articles in recent years. 